\title{
Dying well with dementia: qualitative examination of end-of-life care ${ }^{\dagger}$
}

Vanessa Lawrence, Kritika Samsi, Joanna Murray, Danielle Harari and Sube Banerjee

\section{Background}

People with dementia often die badly, receiving end-of-life care of poorer quality than that given to those who are cognitively intact.

\section{Aims}

To define good end-of-life care for people with dementia and identify how it can be delivered across care settings in the UK.

\section{Method}

In-depth interviews were conducted with 27 bereaved family carers and 23 care professionals recruited from the community, care homes, general hospitals and continuing care units. Data were analysed using the constant comparison method.

\section{Results}

The data highlighted the challenge and imperative of 'dementia-proofing' end-of-life care for people with dementia. This requires using dementia expertise to meet physical care needs, going beyond task-focused care and prioritising planning and communication with families.

\section{Conclusions}

The quality of end-of-life care exists on a continuum across care settings. Together, the data reveal key elements of good end-of-life care and that staff education, supervision and specialist input can enable its provision.

\section{Declaration of interest}

None.
There are 700000 people with dementia in the UK, ${ }^{1}$ and at least 100000 of these die each year. ${ }^{2}$ National policy is clear that people should receive good end-of-life care irrespective of the condition with which they die, ${ }^{3}$ yet evidence suggests that people with dementia and their families receive poor-quality care in the final stages of their lives. ${ }^{4}$ Reported problems include underdiagnosis and poor treatment of pain, ${ }^{5}$ painful and unnecessary investigations, ${ }^{6}$ and inappropriate use of aggressive treatments. ${ }^{7,8}$ It is argued that failure to recognise dementia as a terminal condition, and the costs attached to accommodating non-cancer patients, may preclude access to palliative care. ${ }^{8,9}$ In fact, the majority of deaths of people with dementia occur in institutional settings such as care homes and hospitals, ${ }^{10,11}$ where unmet needs and concerns about care quality appear most pervasive. ${ }^{12}$ In an attempt to pre-specify the content of end-of-life care, advance care planning has emerged. ${ }^{3,13}$ In the USA advance directives, in which a person with dementia can indicate his or her wish to refuse medical treatment, have been associated with reduced family stress. ${ }^{14}$ However, these are used only infrequently in the UK. ${ }^{15}$

It is clear that questions remain regarding the needs and preferences of people with dementia and the adequacy of care practices across settings. ${ }^{16}$ Developing a model of end-of-life care for people with dementia requires an understanding of the priorities and concerns of key stakeholders. ${ }^{17-19}$ By investigating experiences of good and bad end-of-life care in the community, in National Health Service (NHS) continuing care units, in care homes and in general hospitals, we aimed to identify how effective, good-quality end-of-life care might be delivered for people with dementia across care settings.

\section{Method}

\section{Participants}

This qualitative study involved in-depth interviews with care professionals and bereaved carers of people with dementia. The local research ethics committee approved the study. Four

†See editorial, pp. 357-359, this issue. community mental health teams, five care homes, five NHS continuing care units and two general hospitals participated in the research. Researchers introduced the aims and objectives of the study at staff meetings, and care professionals were invited to participate in an interview and to identify and introduce the research to eligible bereaved carers. The researchers emphasised that they were interested in speaking with carers with both positive and negative end-of-life care experiences. Carers were defined as eligible if the person with dementia for whom they had cared had died in the previous 2-6 months. Carers who expressed an interest in hearing more about the study were sent an information sheet, which was followed by a telephone call from the researcher. We also interviewed - and recruited through - care professionals in palliative care teams, liaison psychiatry teams, Alzheimer's Society and carer organisations to explore the full diversity of attitudes, experiences and beliefs. Purposive sampling was conducted across four south London boroughs (Lambeth, Lewisham, Southwark and Croydon) to select participants with a range of characteristics that might be expected to influence experiences and perceptions of good end-of-life care (e.g. professional role, length of employment, relationship to the person with dementia, age). Recruitment continued until saturation; that is, until the devised categories had been fully explored and new data were easily accommodated within them.

\section{Qualitative interviews}

Initial interview guides incorporating topics of interest were generated from the literature. The interviews with carers began by exploring the quality of life of their relative (the person with dementia) in the final 6 months of the latter's life. Carers identified positive and negative aspects of care relating to staff, the care environment, the management of pain and distress, treatment decisions and their relative's death. Carers were also asked to reflect on their relative's care preferences, attitudes towards care planning and what, if anything, they wished they had done differently. Interviews with care professionals examined attitudes towards good end-of-life care, the challenges to its provision and issues surrounding planning, treatment decisions 
and interaction with the family. The interview guides were amended iteratively and carefully followed the participants' concerns. Obtaining the perspectives of informal (family) and formal (paid) carers provided complementary insights into the provision of care. Care professionals described different priorities, strategies and styles when providing end-of-life care, which family carers were able to evaluate. Examining the research question from different angles in this way acted as a form of triangulation. ${ }^{20}$ Interviews lasted for up to an hour and were conducted either in the workplace (for care professionals) or at home (for bereaved carers). The interviews were tape-recorded and transcribed verbatim.

\section{Data analysis}

Three of the researchers (V.L., K.S. and J.M.) read the first five transcripts repeatedly to immerse themselves in the data; they then independently separated the data into meaningful fragments and emerging themes were labelled with codes. Coding strategies were compared and any instances of disagreement were discussed until a consensus was reached. This process helped to highlight alternative interpretations and, alongside interview notes and research team meetings, raise awareness of personal values and preconceptions. One researcher (V.L.) then completed the analysis of the interviews using the constant comparison method to delineate similarities and differences between the codes. ${ }^{21}$ This enabled codes to be grouped together to form higher-level conceptual categories (e.g. 'going beyond task-focused care') which were verified and refined as the analysis proceeded. Ideas about categories, subcategories and their relationships were recorded in theoretical memos, ${ }^{21}$ and regularly discussed in research team meetings. Data are presented across the participant groups and similarities and discrepancies are highlighted where relevant. Quotations used in the text are labelled by type of carer: care professional $(\mathrm{CP})$ or bereaved family carer $(\mathrm{BC})$, job title and place of work (for care professionals), relationship to the person with dementia and place of death of the person with dementia (for bereaved family carers).

\section{Results}

Twenty-three care professionals and 27 bereaved family carers participated in an interview (Table 1). Within the sample, 11 people with dementia had died in an NHS continuing care ward, 5 in a care home, 5 in their own home and 6 in a general hospital. However, most had experience of care in several different settings. Similarly, care professionals expressed opinions on end-of-life care beyond the setting in which they worked. Three elements of good end-of-life care emerged from the data: first, using dementia expertise to meet physical care needs; second, going beyond task-focused care; and third, prioritising planning and communication. Although not specific to people with dementia, the challenge of meeting these needs is amplified among this population.

\section{Meeting physical care needs}

Meeting the physical care needs of people with dementia at the end of life necessitated an understanding of the cognitive, functional and communication problems posed by the condition. A lack of knowledge and expertise in dementia care was manifest in failures to attend to personal care or hygiene, and in care professionals' expressed lack of confidence in addressing palliative care needs such as pain and discomfort. Palliative care specialists emphasised that identifying and responding to the physical care needs of the person with dementia must form the cornerstone of any approach.

'Well people with dementia in the advanced stages actually don't have that many complex needs, it's actually quite basic care needs that are not being met.' (Specialist nurse, palliative care, CP22)

Ensuring adequate food and fluid intake was considered paramount, but care homes were occasionally evaluated negatively in this respect. Some were criticised for providing insufficient support with eating, whereas others were criticised for feeding the person with dementia unnecessarily. Hospitals were condemned for failing to meet this basic need. Numerous examples were given of people with dementia being offered food that they were unable to chew, swallow or cut up. For many, this typified hospital staff's lack of understanding of the needs of people with dementia and what to do to meet them.

'There was no people feeding them and I went, I used to go in and feed her and they said, "Oh no, she's here to be rehabilitated, you shouldn't feed her, it's spoiling her, she can do it herself," well ... . she couldn't do it at all and as I say her eating was getting worse and worse at that stage.' (Daughter, general hospital, BC18)

The provision or absence of good nursing care frequently dominated family carers' accounts. Family carers were highly appreciative when their relative's personal care and hygiene were attended to efficiently: equally, instances (often in general hospitals) in which individuals were not routinely washed, toileted or dressed provoked enormous distress. Staff in almost all settings identified pain control as underlying good-quality end-of-life care for people with dementia; however, it was implied that this was challenging because individuals might be unable to verbalise their discomfort. Continuing care staff and palliative care nurses

Table 1 Characteristics of participating bereaved family carers and care professionals

$n(\%)$

Bereaved carers $(n=27)$

Site of death

care home

community

Continuing care unit

General hospital

5 (18)

Gender

Male

$6(22)$

Female

$6(22)$

$21(78)$

Relationship to the person with dementia Wife

Daughter

$8(30)$

Son

5 (18)

Other family

$5(18)$

Friend

$1(4)$

Care professionals $(n=23)$

Place of work

Alzheimer's Society

Care home

Community mental health team

Continuing care unit

General hospital

Liaison psychiatry

Palliative care

Job title

Assistant psychologist

Care assistant

Clinical nurse specialist

Clinical psychologist

Consultant physician

Deputy manager

Manager

Nurse

Psychiatric nurse

Psychiatrist
$1(4)$

3 (13)

4 (17)

7 (30)

3 (13)

3 (13)

2 (9)

$1(4)$

3 (13)

2 (9)

$1(4)$

1 (4)

3 (13)

4 (17)

3 (13)

2 (9)

3 (13) 
stressed the importance of assessing facial expressions, movements, reactions and changes in the individual when assessing pain - skills developed by working with people with dementia and getting to know individuals. Care professionals working in general hospitals acknowledged that these needs risked being overlooked in the hectic and demanding ward environment.

'You have other distractions and that's as much a challenge, isn't it, that you've got to make sure that person who can't communicate, can't move, can't respond at all, is comfortable and got good mouth care and so on, and yet the acutely unwell person is, you know, we have life saving maybe.' (Matron, general hospital, CP21)

Pain management in care homes was also criticised.

'My experience of going into nursing homes is that I will often think that non-verbally someone is indicating to me that they have got pain, but because the person can't tell the nurse looking after them that they have got pain the nurse doesn't recognise it. (Palliative care nurse, general hospital, CP23)

Few family carers made any reference to palliative care. Professionals in care homes often had limited experience of this service and hospital staff commented that people with dementia were less likely to receive a palliative care referral. Yet staff across the sample discussed the value of a palliative care input. The delivery of novel methods of pain relief, such as syringe drivers and slow-release patches, was identified as one of its principal advantages. Palliative care nurses were considered skilled in identifying and managing pain in patients with complex needs and were also sensitive to nausea and hallucinations in people with dementia at the end of life. Second to pain control was the perceived support and reassurance that palliative nurses offered nursing staff in these settings. Managers and care assistants stated that they found it helpful to know what to expect in the patient's last few days and to be reassured that they were doing the right thing. Continuing care staff often felt equipped to provide endof-life care, yet also valued the option of making a referral to palliative care if necessary. Expertise, and confidence in one's expertise, were considered vital in making difficult treatment decisions, such as withdrawing active treatment. Guidelines such as the Liverpool Care Pathway (www.liv.ac.uk/mcpcil/liverpoolcare-pathway) helped care professionals and family members accept the legitimacy of such action.

I think it has changed people's attitudes and I think it makes sure that somebody, because we think she's dying we shouldn't be bleeding her every day or sticking any tubes in her or that sort of thing. Before there was no structure on how you would look after somebody who's dying on the ward.' (Liaison psychiatrist, general hospital, CP15)

Palliative care nurses commented that the Gold Standards Framework (www.goldstandardsframework.org.uk) was helping their involvement in the care of individuals in the community and care homes. However, there was also recognition that this framework required a substantial financial commitment from care homes and a willingness to release staff for training. Other care homes were not working with these protocols and did not appreciate why they might be of benefit.

\section{Beyond task-focused care}

A wide range of care professionals proposed that functional dependency defined the end of life of people with dementia. Accordingly, and despite the weaknesses in physical care provision outlined above, some felt that meeting physical needs and symptoms defined end-of-life care in this population. One psychologist and two palliative care specialists highlighted the risk of becoming entirely task-focused, noting how important but difficult it is for staff to empathise with the person with dementia in the final stages of the illness. It was felt this was a particular problem in dementia because of cognitive and communication issues, which may even make a person with dementia a lessfavoured patient owing to a lack of reciprocity. Care professionals across settings acknowledged that they sometimes struggled to see that the person was 'still there'.

'We don't know whether someone is still hearing what we are saying. You know, you think only their heartbeat is left.' (Deputy manager, care home, CP18)

It was suggested that concentrating on practicalities rather than on individuals' emotional needs might allow staff to distance themselves from difficult situations. This was epitomised in attitudes towards a good death. Whereas some care professionals focused on providing emotional comfort, others listed the practical tasks that had to be completed at that time.

'We were just preparing, tidying up, making her clean, her face and then sitting her up. All we can do for someone is make sure the room is clean, tidy.' (Deputy manager, care home, CP18)

Care professionals all talked of a holistic approach encompassing the individual's physical, psychological, social, emotional and cultural needs. However, only a minority demonstrated how this was implemented in their everyday work with people with dementia. These professionals argued that physical care must underpin any approach, but that this has be delivered with compassion and tailored to the needs of the individual. This formed the crux of the family carers' accounts and for the most part, end-of-life care was evaluated positively if it was felt that the professionals cared about their dying relative. Families prioritised a 'warm atmosphere' where people with dementia were made to feel relaxed and safe.

'We was just glad that my mum had found this lovely home for him and I would recommend it to anybody because they do care. When he was in his bed they used to tell him what they was going to do, like if he was going to be washed, they would tell him that they was going to wash him ... they talked him all the way through so that he wouldn't get frightened or anything and they were just brilliant with him.' (Cousin, continuing care unit, BC08)

Family members spoke positively about staff interacting with their relative. This emerged as a distinctive feature of the care professionals in the NHS continuing care units and some of the care homes included in this research. Staff described getting to know individual's interests, sensitivities and preferences. A number of NHS continuing care staff stated that assessments were conducted with the patient and their family at admission, which helped to build a portrait of the person. Getting to know the individual's family also provided insight into the patient and helped to make decisions regarding their everyday care. This was identified as a difficulty within general hospitals, as staff did not have the advantage of spending time with the individual and their families over a long period. Getting to know the individual also proved expedient in meeting physical needs, as care professionals were better placed to identify changes in the patient and thus detect suffering or discomfort. Providing individualised care could also avoid distress.

'She would never ever wear skirts, she would wear trousers and jumpers and the staff not knowing that would put her skirt on her and she would get all upset and irate and wasn't able to express that to the staff.' (Community psychiatric nurse, community, CP12)

However, care professionals acknowledged that seeing a person that they cared for dying could be difficult to bear. It was considered a challenge to balance personal and professional feelings, yet these demands often went unrecognised within dementia care.

'I'm just not sure sometimes how much support staff working with people with dementia are given in terms of recognising just how distressing and sad it can be to see people coming to the end of their lives and losing all their independence and losing a lot of their skills and also dealing with the impact on families ... you can't do that unless you are well supported.' (Psychologist, community, CP09)

\section{Planning and communication with family}

Although family members speculated about the respective advantages and disadvantages of advance directives and advance statements, few were aware of their existence prior to the interview 
and the majority felt that their relative's confusion had precluded planning of this sort. Care professionals similarly lacked experience of advance planning and concurred that timing was crucial: introducing the topic too soon after diagnosis might engender distress or despair; too late in the illness and the person with dementia might lack capacity to make such decisions. Only one of the 23 care professionals, manager of a local Alzheimer's Society branch, considered it her responsibility to assume this role. Staff within care homes and NHS continuing care wards felt that this should be discussed around the time of diagnosis, whereas staff within community mental health teams stated that they preferred to be optimistic at that stage. Thus, despite a consensus among care professionals that people with dementia should be given the opportunity to plan for the future, it was apparent that this opportunity might not always arise. It was seen as 'someone else's problem', with family carers unclear as to potential benefits and risks since professionals had not discussed these issues with them. Some speculated that treatment preferences might change, thus undermining the validity of the advance directive. One family member commented that advance care planning might alleviate the burden associated with this role. 'I think it makes it easier for the carer if they know because then you haven't got that moral dilemma. Because like I was placed in . . . was I stopping her having her last chance of life by not letting her go to [hospital] for the dehydration? ... Would she have wanted it? You know you tear yourself in pieces.' (Daughter, general hospital, $\mathrm{BC} 23$ )

Professional and family carers discussed their respective roles in planning end-of-life care and reaching treatment decisions. Distinctive trends emerged across the care settings: a strong emphasis on communication, underpinned by clear procedures for involving family members, was evident in NHS continuing care wards, occasionally present in care homes and largely absent in general hospitals. Staff in NHS continuing care units noted and this was reflected in the comments of family carers - that some preferences were routinely discussed and recorded around the point of admission (e.g. procedures often existed for documenting wishes regarding resuscitation and funeral arrangements). Although some treatment decisions could not be made this far in advance, there was a clear demarcation between care settings that took a proactive rather than reactive approach to end-of-life care. The question of whether individuals should be transferred to hospital during the final stages of their life emerged as one of the most common and problematic decisions. It was evident that hospitalisation was a frequent occurrence despite agreement among care professionals that this was often inappropriate.

'If you take a nursing home patient you know, somebody who isn't eating or drinking, I mean they're dehydrated, they will send them to hospital when actually invariably somebody may die but actually those are the people that we shouldn't see. Those are the people who should remain in nursing homes and they should have an endof-life care plan.' (Nurse, general hospital, CP21)

A distinguishing feature of NHS continuing care wards and certain care homes was the attention given to discussing this issue with families and the wider care team. Meetings were often scheduled to provide family members with information and to reach a unified position. Other treatment decisions covered the use of feeding tubes, catheters, antibiotics, investigative procedures and interventions. Some relatives complained that hospital staff neither informed nor consulted them about these issues. Palliative care staff noted that professionals across care settings could be reluctant to withdraw active treatment in the absence of explicit planning or a clear consensus among the care team.

'He was imminently dying, yet the [general practitioner] phoned the family and I think the way it was put to the family, well, the family then said let's send him to hospital, I don't know if the family actually realised that he was imminently dying or what they thought hospital would achieve. So it was quite distressing for me and the staff to see this man who was imminently dying being shipped off to hospital.' (Nurse specialist, palliative care, CP22)
Critically, achieving a good death also involved enabling family members to be present at the time of death. There was consensus that this was of enormous importance for both the person with dementia and the family. If this was not possible, either because the individual lacked close family members or had deteriorated unexpectedly, continuing care staff noted that they would assume the role of 'being there' themselves. Notably, three of the five people with dementia who had died in the community had done so unexpectedly, either of a heart attack or peacefully in their sleep. Two family members who planned for their relative to die at home demonstrated exceptional organisational skills and determination in ensuring this happened.

'We knew she wanted to be at home so we put in place arrangements ... we were talking about palliative care and that sort of thing with local authorities ... and things like the carers, for example, we took them into the hospital, the administrators from the care company, to be with the nurse who showed how to actually feed her, to put some water in her mouth and how to actually handle her. And we had got the hospital bed in, we turned the dining room into the bedroom, we got a big hospital bed, we got a hoist, we got all the equipment in and the carer administrator came on the first day to do the risk assessment.' (Son, community, BC14)

\section{Discussion}

The study's primary finding was that there is a need to 'dementiaproof' end-of-life care for people with dementia. If end-of-life care does not take into account the unique circumstances and needs of people with dementia, it is likely to fail them. This requires service providers and care professionals to ensure that the environments in which people live and die - be they at home, in a care home, in NHS continuing care or in a general hospital - do three things: use knowledge of dementia to identify and respond to physical care needs; go beyond taskfocused care; and prioritise planning and communication with the family.

The data support the suggestion that NHS continuing care units might act as a model for meeting the complex needs of people with advanced dementia. ${ }^{17}$ The units in this study provided valuable examples of good end-of-life care, whereby care plans were carefully formulated with the family and services worked to ensure that they were followed, including the avoidance of transfer to acute hospitals. The care homes within this study were in an intermediate position with respect to quality; some homes, where basic and palliative care needs had been identified and met and staff had got to know the individual, provided positive end-of-life experiences equivalent to those in NHS continuing care units. However, where there was less communication with the family or where plans were not followed and there were admissions to acute hospitals, individuals had poor experiences. For people with dementia we found that death in general hospital was almost invariably associated with poor quality, with staff appearing to provide inadequate assistance with eating and drinking, and failing to manage pain, to seek information from carers about the individual or to discuss treatment options with families at the end of life. A high proportion of people with dementia in the UK die in general hospitals, ${ }^{11}$ and improvement in end-of-life care and in general hospital care are both priorities in the National Dementia Strategy, ${ }^{13}$ but the data indicate that little as yet has been achieved. Notably, few care professionals or relatives claimed experience of providing end-of-life care in the community, which is consistent with the small proportion of people with dementia who die at home. ${ }^{11}$ That three of these five family members had not planned for this event further clarifies the need for community practitioners to explore preferences for place of death with individuals and their families. ${ }^{22}$

The data highlight that basic care needs are not uniformly met and that this causes considerable distress. Failure to attend to 
personal care and hygiene or to ensure adequate food and fluid intake appeared to reflect the decline in the person with dementia's ability to communicate wishes and needs. ${ }^{23}$ It is vital that care professionals have an understanding of dementia and are alert to the specific needs of this population. Staff interviews tended to focus on the specialist nursing skills that are required at end-of-life care, and although controlling pain and other symptoms is integral to a palliative care approach, ${ }^{24}$ this must not overshadow the imperative of meeting basic nursing needs. Moreover, the data corroborate suggestions that healthcare professionals can, despite the priority afforded to this area, lack the necessary skills to control pain and discomfort in the person with dementia. ${ }^{5,25}$ Only palliative care specialists and continuing care staff described using skills acquired in working with people with dementia to assess facial expressions, movements and changes in the individual. Access to palliative care services was limited in this as in other studies. ${ }^{25}$ However, where available, specialist palliative input from staff knowledgeable in dementia provided valuable instruction and support, helping to instil staff with the confidence to manage end-of-life care themselves. This supports data from the St Christopher's Hospice Palliative Care in Dementia Project, an initiative aimed at providing specialist palliative care to people with severe dementia and caregivers, which concluded that most people with dementia could be managed within a 'supported generalised environment. ${ }^{26}$

The provision of psychosocial support is a fundamental tenet of end-of-life care, ${ }^{27}$ and family members were unequivocal in the value that they placed on it. However, the data reinforce suggestions that symptom management can predominate for people with dementia in certain care settings. ${ }^{28}$ Whereas other end-of-life research has described relationships of emotional closeness between staff and residents in long-term care, ${ }^{19}$ it was widely acknowledged that it could be difficult to empathise with people in the advanced stages of dementia. This is likely to inhibit the provision of palliative care, or indeed person-centred care, aimed at addressing physical, social, spiritual and psychological needs. ${ }^{24}$ Yet the data also provided examples of holistic care, characterised by an emphasis on 'getting to know the person', which staff achieved through routine assessments and spending time with the individual and their family. This philosophy appeared embedded in NHS continuing care units, but was largely absent from general hospitals. This may reflect the time constraints within which hospital staff work, but also differential understanding and skills in communicating with people with dementia. Finally, staff commented that forming a close relationship with people with dementia in the final stages of their lives could be deeply distressing. Clearly, care professionals require support in managing the anxieties that can arise; it is no more acceptable to overlook the needs of staff working with people with advanced dementia than it is to deprive people with dementia of appropriate palliative care. $^{29}$

Advance care planning, enabling the person with dementia to articulate care preferences for the future, is a key element of both the End of Life Care Strategy and the National Dementia Strategy. ${ }^{3,13}$ Our findings are indicative of logistical and ethical concerns that have been raised in the literature, including uncertainty around when to discuss care planning and whether one can make a decision for an unknown future self. ${ }^{30}$ The reluctance among care professionals to assume responsibility for discussing advance directives was striking and in line with previous research. ${ }^{31}$ This highlights the necessity of research to examine whether the concerns of care professionals and carers are shared by people with dementia, and whether there is positive value in discussing end-of-life care issues as early as at the point of diagnosis. Such research is needed to formulate guidelines and training to clarify professional roles in this process. Finally, it is evident that the decision to replace active treatment with palliative care can be difficult to make, ${ }^{32}$ and raises practical, ethical and emotional issues that necessitate an active dialogue between the care team and family carers. ${ }^{33}$ A commitment to seeking consensus on these issues emerged as key indicator of good practice. Again, it was staff in NHS continuing care units who were most likely to negotiate with family members in this way. It should be possible to apply the good practice evident in these units in this study to care homes, where a third of people with dementia live.

\section{Strengths and limitations}

The sample size was relatively large for a qualitative study, yet it cannot be assumed that the findings are typical of all care homes, NHS continuing care units, general hospitals or community care across the UK. That said, the diversity that emerged in philosophy and practice, combined with the evaluative comments of family carers, helped to distil properties of good end-of-life care that are likely to have applicability beyond the context of this research.

Combining the perspectives of bereaved family carers and care professionals is a unique feature of this research; that these experiences were drawn from carers and care professionals in different care settings further enhances the novelty of the study.

\section{Future research}

Understanding the priorities and concerns of these groups remains a fundamental first step in developing an appropriate model of end-of-life care for people with dementia. However, we are aware that the paper does not cover the experience of people with dementia who lack close family members. These individuals bring into clear focus the value of advance care plans and it will be important to include their perspectives and those of other people with dementia in future research.

\section{Vanessa Lawrence, PhD, Section of Mental Health and Ageing, Health Service and Population Research Department, Institute of Psychiatry, King's College London; Kritika Samsi, PhD, King's College London, Melbourne House, Aldwych, London; Joanna Murray, BA, Section of Mental Health and Ageing, Health Service and Population Research Department, Institute of Psychiatry, King's College London; Danielle Harari, MD, St Thomas' Hospital, London; Sube Banerjee, MD, Section of Mental Health and Ageing, Health Service and Population Research Department, Institute of Psychiatry, King's College London, UK \\ Correspondence: Sube Banerjee, Institute of Psychiatry, King's College London, De Crespigny Park, London SE5 8AF, UK. Email: s.banerjee@kcl.ac.uk}

First received 9 Mar 2011, final revision 7 Jun 2011, accepted 16 Jun 2011

\section{Funding}

The study was funded by a grant from The Maudsley Charity.

\section{Acknowledgements}

We thank all the bereaved family carers who took part in this research. Participants were generous with their time and often spoke about difficult personal issues in order to describe their experience. The same thanks are due to the care professionals who contributed their time and expertise. We would also like to thank care professionals for their help in recruiting participants to the study.

\section{References}

1 Knapp M, Prince M, Albanese E, Banerjess S, Dhanasiri S, Fernandez J, et al. Dementia UK. Alzheimer's Society, 2007.

2 Bayer A. Death with dementia - the need for better care. Age Ageing 2006; 35: 101-2. 
3 Department of Health. End of Life Care Strategy - Promoting High Quality Care for All Adults at the End of Life. Department of Health, 2008.

4 Ballard C, Fossey J, Chithramohan R, Howard R, Burns A, Thompson P, et al. Quality of care in private sector and NHS facilities for people with dementia: cross sectional survey. BMJ 2001; 323: 426-7.

5 Scherder E, Oosterman J, Swaab D, Herr K, Ooms M, Ribbe M, et al. Recent developments in pain in dementia. BMJ 2005; 330: 461-4.

6 Mitchell SL, Morris JN, Park PS, Fries BE. Terminal care for persons with advanced dementia in the nursing home and home care settings. J Palliat Med 2004; 7: 808-16.

7 Evers MM, Purohit D, Perl D, Khan K, Marin DB. Palliative and aggressive end-of-life care for patients with dementia. Psychiatr Serv 2002; 53: 609-13.

8 Sampson EL, Gould V, Lee D, Blanchard MR. Differences in care received by patients with and without dementia who died during acute hospital admission: a retrospective case note study. Age Ageing 2006; 35: 187-9.

9 Field $\mathrm{D}$, Addington-Hall J. Extending specialist palliative care to all? Soc Sci Med 1999; 48: 1271-80.

10 Mitchell SL, Teno J, Miller SC, Mor V. A national study of the location of death for older persons with dementia. J Am Geriatr SOC 2005; 53: 299-305.

11 Kay DWK, Forster DP, Newens AJ. Long-term survival, place of death, and death certification in clinically diagnosed pre-senile dementia in northern England: follow-up after 8-12 years. Br J Psychiatry 2000; 177: 156-62.

12 Teno J, Clarridge BR, Casey V, welch L, Wetle T, Shield R, et al. Family perspectives on end-of-life care at the last place of care. JAMA 2004; 291: 88-93.

13 Department of Health. Living Well With Dementia: A National Dementia Strategy. Department of Health, 2009

14 Engel SE, Kiely DK, Mitchell SL. Satisfaction with end-of-life care for nursing home residents with advanced dementia. J Am Geriatr Soc 2006; 54: 1567-72.

15 Roger KS. A literature review of palliative care, end of life, and dementia. Palliat Support Care 2006; 4: 295-303.

16 Birch D, Draper J. A critical literature review exploring the challenges of delivering effective palliative care to older people with dementia. J Clin Nurs 2008; 17: 1144-63.

17 Hughes JC, Robinson L, Volicer L. Specialist palliative care in dementia: specialised units with outreach and liaison are needed. BMJ 2005; 330 57-8.

18 Hughes JC, Jolley D, Jordan A, Sampson EL. Palliative care in dementia: issues and evidence. Adv Psychiatr Treat 2007; 13: 251-60.
19 Munn JC, Dobbs D, Meier A, Williams HB, Zimmerman S. The end-of-life experiences in long-term care: five themes identified from focus groups with residents, family members, and staff. Gerontologist 2008; 4: 485-94.

20 Mays N, Pope C. Qualitative research in health care: assessing quality in qualitative research. BMJ 2000; 320: 50-2.

21 Glaser B. Theoretical Sensitivity: Advances in the Methodology of Grounded Theory. Sociology Press, 1978.

22 Munday D, Petrova M, Dale J. Exploring preferences for place of death with terminally ill patients: a qualitative study of experiences of general practitioners and community nurses in England. BMJ 2009; 339 b2391.

23 Shuster JL. Palliative care for advanced dementia. Clin Geriatr Med 2000; 16: 373-86.

24 Small N. Living well until you die. Quality of care and quality of life in palliative and dementia care. Ann NY Acad Sci 2007; 1114: 194-203.

25 Lloyd-Williams M. An audit of palliative care in dementia. Eur J Cancer Care 1996; 5: 53-5

26 Scott S, Pace V. The first 50 patients: a brief report on the initial findings from the Palliative Care in Dementia Project. Dementia 2009; 8: 435-41.

27 National Council for Palliative Care. Palliative Care Bill: Briefing for Peers. National Council for Palliative Care, 2007.

28 Werth JL, Gordon JR, Johnson RR. Psychosocial issues near the end of life. Aging Ment Health 2002; 6: 402-12.

29 Sampson EL, Ritchie CW, Lai R, Raven PW, Blanchard MR. A systematic review of the scientific evidence for the efficacy of a palliative care approach in advanced dementia. Int Psychogeriatr 2005; 17: 31-40.

30 Bisson Jl, Hampton V, Rosser A, Holm S. Developing a care pathway for advance decisions and powers of attorney: qualitative study. Br J Psychiatry 2009; 194: 55-61.

31 Hawkins NA, Ditto PH, Danks JH, Smucker WD. Micromanaging death: process preferences, values and goals in end-of-life medical decision making. Gerontologist 2005; 45: 107-17.

32 Hinkka H, Kosunen E, Lammi U-K, Metsanoja R, Puustelli A, Kellokumpu-Lehtinen $\mathrm{P}$. Decision making in terminal care: a survey of Finnish doctors' treatment decisions in end-of-life scenarios involving a terminal cancer and a terminal dementia patient. Palliat Med 2002; 16: 195-204.

33 Winzelberg GS, Patrick DL, Rhodes LA, Deyo RA. Opportunities and challenges to improving end-of-life care for seriously ill elderly patients: a qualitative study of generalist physicians. J Palliat Med 2005; 8: 291-9. 